\title{
Introdução de alimentos e excesso de peso em pré-escolares de uma comunidade vulnerável da cidade do Rio de Janeiro - Associação da introdução de alimentos e excesso de peso
}

\section{Complementary feeding and overweight among preschoolers from a vulnerable community in Rio de Janeiro city - Association of complementary feeding with overweight}

Maria Beatriz Trindade de Castro'

Débora Souza Gigantel

Luciana de Oliveira Silva'

Bruno Cardoso do Nascimento ${ }^{2}$

Patrícia de Carvalho Padilha ${ }^{3}$

I Departamento de Nutrição Social e Aplicada, Instituto de Nutrição Josué de Castro. Universidade Federal do Rio de Janeiro. Rio de Janeiro-RJ, Brasil.

\section{${ }^{2}$ Clínica da Família Santa Marta. Estratégia Saúde da Família. Secretaria Municipal de Saúde e Defesa Civil da Cidade do Rio de Janeiro. Rio de Janeiro-RJ, Brasil.}

${ }^{3}$ Departamento de Nutrição e Dietética. Instituto de Nutrição Josué de Castro. Universidade Federal do Rio de Janeiro. Rio de Janeiro-RJ, Brasil.

Correspondência /Correspondence Maria Beatriz Trindade de Castro Universidade Federal do Rio de Janeiro Observatório de Epidemiologia Nutricional Av. Carlos Chagas Filho 373, Bloco J -

$2^{0}$ andar - sala 029

21941-590 - Ilha do Governador,

Rio de Janeiro, RJ, Brasil

E-mail:mbtcastro@gmail.com

\section{Resumo}

Objetivo: Descrever a introdução de alimentos no primeiro ano de vida e verificar sua associação e do aleitamento materno com o índice de massa corporal (IMC) para idade entre préescolares de uma creche de uma comunidade vulnerável do Rio de Janeiro. Métodos: Estudo transversal com informações sobre 132 crianças com idade de 2-5 anos matriculadas em uma creche comunitária. O IMC para idade foi aplicado para rastrear excesso de peso. Adotou-se o ponto de corte do escore-z +2 , como proposto pelo protocolo do SISVAN/MS. Empregaram-se os testes $t$-Student e qui-quadrado para avaliar a distribuição das variáveis antropométricas e sociodemográficas segundo o estado nutricional dos pré-escolares. Utilizou-se a regressão linear múltipla para testar os efeitos do aleitamento materno exclusivo, da introdução de alimentos e do escore de alimentação complementar sobre o IMC para idade. Os modelos foram ajustados para idade e sexo do pré-escolar e idade materna. Resultados: A média de idade das crianças foi de 3,3 $( \pm 0,91)$ anos, e $36(28,6 \%)$ pré-escolares apresentaram excesso de peso, mas não houve diferença significativa entre os sexos $(p=0,195)$. Crianças que receberam aleitamento materno exclusivo apresentaram menores prevalências de excesso de peso $(p=0,028)$. Verificouse associação positiva do IMC para idade com o consumo de mingau $(\beta=0,0080 ;$ IC95\% $=0003-0,015 ; p=0,043)$ e negativa $(\beta=-0,001 ;$ IC95\% $=-0,002--0003 ; p=0,016)$ com o escore de alimentação complementar. Conclusão: A introdução precoce 
de alimentos pode contribuir para o risco de excesso de peso. Verificou-se associação do aleitamento materno com menor prevalência de sobrepeso.

Palavras-chaves: Alimentação Complementar. Nutrição Infantil. Aleitamento Materno. Sobrepeso. Índice de Massa Corporal.

\section{Abstract}

Objective: To describe the introduction of foods in the first year of life and verify its association with Body Mass Index (BMI) for Age among preschool children at a daycare center in a vulnerable community in Rio de Janeiro city, Brazil. Methods: Cross-sectional study with 132 children aged between 2-5 years. The classification of overweight was based on BMI for age (z-score +2$)$, as proposed by SISVAN/MS. The frequencies of anthropometric and sociodemographic variables among nutritional status were evaluated by $t$-Student's test and qui-square test. Multiple linear regression was applied to test the association of breastfeeding, introduction of food and complementary feeding score with BMI for age. Models were adjusted by age and sex of infants and mother's age. Results: The mean age of children was $3.3( \pm 0.91)$ years old and $36(28.6 \%)$ were overweight, but no significant difference was found between genders $(p=0.195)$. Exclusively breastfeeding was associated with lower prevalence of overweight $(p=0.028)$. There was positive association between BMI and consumption of porridge $(\beta=0.0080,95 \% \mathrm{CI}=0003-0.015, \mathrm{p}=0.043)$ and negative association with complementary feeding score $(\beta=-0.001$, $95 \%$ CI -0.002 - 0.003, p = 0.016). Conclusion: Early introduction of foods may contribute to the risk of excess of body weight. There was association of breastfeeding and lower prevalence of overweight.

Key words: Supplementary Feeding. Infant Nutrition. Breastfeeding. Overweight. Body Mass Index.

\section{Introdução}

O aleitamento materno pode ser considerado como o primeiro hábito alimentar saudável. ${ }^{1}$ $\mathrm{O}$ leite materno atende às necessidades nutricionais nos primeiros seis meses de vida e possui adequada composição de macronutrientes, além de ser fácil digestão. ${ }^{2}$ Dentre outros benefícios, o leite materno protege contra infecções, evita a ocorrência de quadros diarreicos e protege contra 
doenças respiratórias e alérgicas. Estudos nacionais ${ }^{3-5}$ e internacionais ${ }^{6,7}$ vêm apontando, também, o efeito protetor do aleitamento materno sobre o excesso de peso na infância.

O Ministério da Saúde ${ }^{2}$ recomenda o aleitamento materno exclusivo nos seis primeiros meses de vida, e complementado até os dois ou três anos de idade. De acordo com a II Pesquisa de Prevalência de Aleitamento Materno, ${ }^{8}$ realizada no período de 1999 a 2008, a duração mediana do aleitamento materno exclusivo (AME) aumentou de 23,4 dias para 54,1 dias nas capitais brasileiras e no Distrito Federal. O aleitamento materno complementar (AM) aumentou de 295,9 dias para 341,6 dias no mesmo período.

No Brasil, apesar dos avanços, ainda somente $41 \%$ das crianças se beneficiam do AME até os seis meses de idade. ${ }^{8}$ Associada à baixa prevalência do AME, existe a introdução precoce de água, chás, sucos, outros leites e alimentos. Segundo a literatura científica, ${ }^{3,5}$ a introdução precoce da alimentação associa-se ao excesso de peso e, de forma inadequada, pode repercutir negativamente na saúde em curto e longo prazos. ${ }^{9,10}$

Em estudo transversal realizado por Simon et al. ${ }^{5}$ em São Paulo, com 566 pré-escolares com idade entre dois e seis anos, verificou-se que a introdução de açúcar, dentre outros alimentos, é fator de risco para sobrepeso e obesidade. Segundo dados apresentados por Balaban et al., ${ }^{3}$ a partir de inquérito com 409 pré-escolares de creches conveniadas com a prefeitura da cidade de Recife, o sobrepeso foi mais prevalente entre as crianças que não receberam aleitamento materno exclusivo por até quatro meses.

E ainda, o desmame precoce pode ser inadequado do ponto de vista nutricional. ${ }^{8}$ Caetano et al. ${ }^{11}$ verificaram que, dentre as crianças que recebiam mamadeira aos três meses de idade, somente $34,7 \%$ recebiam fórmula infantil com preparo adequado. Em relação à alimentação das crianças de seis a 12 meses de idade, os autores verificaram baixa ingestão de micronutrientes como ferro, niacina, vitamina E e Zinco. Nesse grupo, evidenciou-se também elevado percentual de consumo de alimentos industrializados, refrigerantes e sucos artificiais.

A alimentação complementar adequada, em paralelo ao aleitamento materno, é de suma importância para o crescimento e desenvolvimento da criança, e para sua saúde na vida adulta. Cabe aos nutricionistas, profissionais da saúde e áreas afins, promoverem a introdução de alimentos saudáveis e monitorarem o estado nutricional das crianças nos primeiros anos de vida. Assim, o objetivo do presente estudo foi descrever a introdução de alimentos no primeiro ano de vida e verificar sua associação e do aleitamento materno com o índice de massa corporal para idade entre pré-escolares de uma creche de uma comunidade vulnerável do Rio de Janeiro. 


\section{Metodologia}

Desenho e População de Estudo

Foi realizado estudo transversal com dados antropométricos e do consumo alimentar de crianças pré-escolares com idade entre dois e cinco anos, matriculadas em uma creche localizada na comunidade do Santa Marta, no bairro de Botafogo, Rio de Janeiro. Todas as 132 crianças préescolares matriculadas na creche foram consideradas elegíveis para o estudo. Desse total, a amostra contou com $126(95,5 \%)$ pré-escolares que foram observados quanto ao peso e a estatura e com 96 (72,7\%) indivíduos que responderam ao questionário contendo informações sociodemográficas e sobre o aleitamento materno e introdução dos alimentos no primeiro ano de vida.

\section{Coleta de Dados}

O estudo deu origem ao projeto denominado Alimentação Saudável e Higiene Bucal: Parceria que dá Gosto, uma parceria entre os profissionais da Estratégia Saúde da Família da Clínica da Família (CF) Santa Marta com a Universidade Federal do Rio de Janeiro. A coleta de dados periódica com os pré-escolares foi realizada durante o estágio curricular das alunas matriculadas no oitavo período do curso de graduação de Nutrição, do Instituto de Nutrição Josué de Castro da Universidade Federal do Rio de Janeiro.

\section{Informações Antropométricas}

Os dados antropométricos coletados foram estatura $(\mathrm{cm})$ e peso $(\mathrm{kg})$. A estatura foi aferida com o emprego do estadiômetro portátil da marca Alturaexata. Nessa ocasião, as crianças encontravamse eretas, com os braços estendidos ao longo do corpo e com as cabeças livres de adereços. As crianças foram pesadas sem sapatos e sem casaco, ou qualquer outro adereço, e posicionadas no centro da balança digital portátil da marca Plena para $150 \mathrm{~kg}$ e precisão $0,1 \mathrm{~kg}$. Ambas as medidas antropométricas foram obtidas de acordo com a metodologia recomendada por Lohman et al. ${ }^{12}$ 


\section{Análise dos Dados}

\section{Desfecho}

A partir das medidas do peso e da estatura, calculou-se o índice de massa corporal [IMC= Peso $(\mathrm{kg}) /$ estatura $^{2}$ (metro)]. A classificação do estado nutricional foi realizada com base no IMC para idade proposto pela Organização Mundial da Saúde, ${ }^{13}$ definindo-se como excesso de peso (sobrepeso e obesidade) valores superiores ao escore- $z+2$.

\section{Variáveis Explicativas}

Durante a coleta de dados, foi enviado aos responsáveis dos pré-escolares, através da agenda escolar, um questionário estruturado com questões sobre: 1) aleitamento materno: recebeu aleitamento materno exclusivo ( $\operatorname{sim}=0$ ou não $=1$ ), a criança ainda mama no peito (sim ou não), até que idade a criança mamou no peito (meses); e 2) introdução de alimentos no primeiro ano de vida: suco/fruta; mamadeira, legumes, sopa, mingau, comida caseira, refrigerante, biscoito, doces/balas, comida tipo fast-food. Para cada item da alimentação complementar, a mãe/responsável poderia indicar o período no qual o alimento foi introduzido: antes de 2 meses; entre 2 e 4 meses; entre 4 e 6 meses; e depois dos seis meses de idade.

\section{Covariáveis sociodemográficas}

Foram consideradas como covariáveis sociodemográficas as informações obtidas também através do questionário: idade da criança (anos), idade materna (anos), escolaridade materna (última série que cursou com aprovação), emprego atual (sim ou não), tipo de trabalho (fixo, informal/autônomo ou outro), situação marital (casada/união estável, solteira ou outro) e número de filhos (número).

Todos os responsáveis foram informados e esclarecidos sobre a realização da pesquisa e quanto ao levantamento das informações sobre a alimentação dos pré-escolares no primeiro ano de vida. O preenchimento do questionário foi condicionado a participação voluntária e assinatura do Termo de Consentimento Livre e Esclarecido pela(s) mãe(s) ou responsável(is) da(s) criança(s). O estudo foi planejado respeitando-se os aspectos éticos previstos pela legislação brasileira, e aprovado pelo Comitê de Ética em Pesquisa da Prefeitura do Rio de Janeiro (Protocolo do CEP SMSDC-RJ: № 125/12). 


\section{Análise Estatística}

A análise estatística foi realizada em quatro etapas: i) caracterização da amostra de pré-escolares segundo as covariáveis antropométricas e sociodemográficas (tabela 1); ii) distribuição do estado nutricional dos pré-escolares (com ou sem excesso de peso) segundo as covariáveis antropométricas e sociodemográficas e as variáveis explicativas (aleitamento materno exclusivo e introdução dos alimentos no primeiro ano de vida); iii) teste da associação das covariáveis antropométricas e sociodemográficas com o desfecho (IMC para idade) e com as variáveis explicativas; e iv) análise do efeito das variáveis explicativas sobre o IMC para idade.

Tabela 1. Características antropométricas e sociodemográficas de 126 crianças pré-escolares matriculadas em uma creche de uma comunidade vulnerável na cidade do Rio de Janeiro-RJ, 2012.

\begin{tabular}{lcc}
\hline Variáveis & Média & $( \pm \mathrm{DP})$ \\
\hline Antropométricas & 15,9 & \\
Peso $(\mathrm{kg})$ & 99,3 & $(3,10)$ \\
Estatura $(\mathrm{cm})$ & 15,9 & $(7,82)$ \\
IMC $\left(\mathrm{kg} / \mathrm{m}^{2}\right)$ & & $(3,01)$ \\
Sociodemográficas & 3,3 & \\
Idade $($ anos) & 29,1 & $(0,91)$ \\
Idade materna (anos) & 8,1 & $(6,59)$ \\
Escolaridade materna (série) & 2,0 & $(3,05)$ \\
No de filhos $(n)$ & No & $(1,16)$ \\
\hline & & $(\%)$ \\
\hline Antropométricas & 36 & $(28,6)$ \\
Excesso de Peso & 90 & $(71,4)$ \\
$\quad$ Sim & & \\
\hline
\end{tabular}


Para avaliar a distribuição das variáveis antropométricas e sociodemográficas, e das variáveis explicativas segundo o estado nutricional dos pré-escolares, foram empregados os testes $t$-Student e qui-quadrado, respectivamente, para analisar dados contínuos e categóricos (tabela 2). Em seguida, a análise de regressão linear univariada foi empregada para testar a associação de cada covariável sociodemográfica com o desfecho, IMC para idade (tabela 3). Todas as covariáveis que apresentaram valores de $p \leq 0,20$ foram consideradas como variáveis de ajuste na análise de regressão linear múltipla subsequente. A análise de regressão linear múltipla testou o efeito do aleitamento materno exclusivo e da introdução de alimentos no primeiro ano de vida sobre o IMC para idade.

Tabela 2. Características antropométricas, sociodemográficas, aleitamento materno e introdução de alimentos segundo o estado nutricional (sem excesso de peso $<$ escore-z +2 e com excesso de peso $\geq$ escore-z +2 ) de 126 crianças pré-escolares matriculadas em uma creche de uma comunidade vulnerável na cidade do Rio de Janeiro-RJ, 2012.

\begin{tabular}{|c|c|c|c|c|c|}
\hline \multirow[t]{3}{*}{ Variável } & \multicolumn{4}{|c|}{ Excesso de Peso } & \\
\hline & \multicolumn{2}{|c|}{$\begin{array}{c}\text { Não } \\
(\mathrm{n}=90)\end{array}$} & \multicolumn{2}{|c|}{$\begin{array}{c}\text { Sim } \\
(\mathrm{n}=36)\end{array}$} & \\
\hline & \multicolumn{4}{|c|}{ Média ( $\pm \mathrm{DP})$} & valor de $p$ \\
\hline \multicolumn{6}{|l|}{ Antropométricas } \\
\hline Peso $(k g)$ & 15,1 & $(2,29)$ & 17,9 & $(3,93)$ & $<0,001$ \\
\hline Estatura $(\mathrm{cm})$ & 99,2 & $(7,19)$ & 99,5 & $(9,30)$ & 0,834 \\
\hline \multicolumn{6}{|l|}{ Sociodemográficas } \\
\hline Idade (anos) & 3,3 & $(0,90)$ & 3,2 & $(0,96)$ & 0,540 \\
\hline Idade materna (anos) & 28,5 & $(6,39)$ & 30,6 & $(6,98)$ & 0,190 \\
\hline Escolaridade materna (série) & 8,1 & $(2,94)$ & 8,1 & $(3,36)$ & 0,898 \\
\hline № de filhos $(n)$ & 2,0 & $(1,25)$ & 2,0 & $(0,94)$ & 0,758 \\
\hline
\end{tabular}




$$
\mathrm{n} \underline{\mathrm{o}}(\%)
$$

valor de $p$

\section{Sociodemográficas}

Sexo do Pré-escolar

Masculino
Feminino

Tipo de Trabalho Materno

Formal

Informal

Estado Civil Materno

Casada/União

Solteira/Outro

\section{Aleitamento Materno}

Exclusivo

Sim

Não

\section{Introdução de Alimentos ${ }^{\ddagger}$}

Frutas/Suco

$$
\begin{aligned}
& <4 \text { meses } \\
& >4 \text { meses }
\end{aligned}
$$

Legumes

$<4$ meses

$>4$ meses

Mamadeira

$<4$ meses

$>4$ meses

Mingau

$<4$ meses

$>4$ meses

Sopa

$<4$ meses

$>4$ meses

Comida Caseira

$$
\begin{aligned}
& <4 \text { meses } \\
& >4 \text { meses }
\end{aligned}
$$

Biscoito

$$
\begin{aligned}
& <4 \text { meses } \\
& >4 \text { meses }
\end{aligned}
$$

57

07

49

41

$(76,6)$

15

21

$(23,4)$

$(33,9)$

0,785

$42 \quad(75,0) \quad 14$

$14 \quad(25,0)$

10

$(71,4)$

04

$(28,6)$

40

24

$(71,4)$

$(68,6)$

16

11

$(28,6)$

$(31,4)$

0,772

0,028

$(25,0)$

$(53,3)$

19

08

1,000

23

$(71,9)$

09

$(28,1)$

46

$(71,9)$

18

$(28,1)$

17

51

$(70,8)$

07

20

28

$(68,3)$

13

11

$(31,7)$

$25 \quad(69,4)$

$(30,6)$

0,263

$20 \quad(80,0)$

05

$(20,0)$

$38 \quad(67,9)$

18

$(32,1)$

0,678

$16 \quad(69,6)$

07

$(30,4)$

$54 \quad(74,0)$

19

$(26,0)$

0,715

$\begin{array}{ll}06 & (66,7) \\ 63 & (72,4)\end{array}$

03

24

$(33,3)$

$(27,6)$

0,716

0,923

0,913

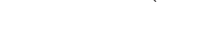

07

$(77,8)$

02

$(22,2)$

62

$(72,1)$

$(27,9)$

Teste $t$-Student para médias e Chi-square para proporção. ${ }^{\dagger}$ Não houve introdução de fast food antes dos 4 meses de idade. Houve um caso de oferta de refrigerante e um caso de oferta de doce antes dos 4 meses de idade. 
Tabela 3. Regressão linear univariada do índice de massa corporal para idade e variáveis sociodemográficas. Rio de Janeiro-RJ, 2012.

\begin{tabular}{lcccc}
\hline \multicolumn{1}{c}{ Variáveis } & $B$ & $\mathrm{IC} 95 \%$ & $\mathrm{R}^{2}$ & Valor de $p$ \\
\hline Idade (anos) & 0,703 & $0,554-0,852$ & 0,413 & $<0,001$ \\
Idade materna (anos) & 0,040 & $0,014-0,065$ & 0,096 & 0,003 \\
Escolaridade materna (série) & 0,019 & $-0,040-0,079$ & 0,005 & 0,517 \\
№ de filhos $(n)$ & $-0,002$ & $-0,155-0,152$ & 0,000 & 0,984 \\
Sexo do pré-escolar & 0,097 & $-0,257-0,451$ & 0,002 & 0,588 \\
Tipo de trabalho materno & $-0,117$ & $-0,527-0,294$ & 0,004 & 0,574 \\
Estado civil materno & $-0,073$ & $-0,350-0,205$ & 0,003 & 0,604 \\
\hline
\end{tabular}

O aleitamento materno foi categorizado em $\operatorname{sim}(1=$ recebeu aleitamento materno exclusivo) e não (zero= não recebeu aleitamento materno exclusivo).

A introdução de alimentos no primeiro ano de vida foi testada pelo mês de introdução de cada um dos alimentos contidos no questionário estruturado (suco/fruta; mamadeira, legumes, sopa, mingau, comida caseira, refrigerante, biscoito, doces/balas, comida tipo fast-food) e pela introdução global desses alimentos, a partir de um escore da alimentação complementar.

Para cada alimento introduzido no primeiro ano de vida, foi gerada uma variável binária (1= introdução antes dos 4 meses de idade e 0 = introdução após os 4 meses de idade). Como não houve introdução de alimentos do tipo fast-food e ocorreu apenas um caso de oferta de refrigerante e doces antes dos quatro meses, o efeito desses alimentos sobre o IMC para idade não foi analisado.

Para a avaliação global da introdução dos alimentos no primeiro ano de vida, gerou-se um escore da alimentação complementar. Exceto pelos alimentos mencionados anteriormente (tipo fast-food, refrigerante e doces), computou-se um valor referente ao mês da introdução de cada um dos alimentos ( $1=$ antes dos 2 meses; $2=$ entre 2 e 4 meses; $3=$ entre 4 e 6 meses; $4=$ após os 6 meses), que foi multiplicado por dois, e a soma de todos os alimentos foi multiplicada por 100, ou seja, adotou-se a seguinte equação: [(Frequência da introdução de Frutas/Suco*2) + (Frequência da introdução de Legumes*2) + (Frequência da introdução de Mamadeira*2) + (Frequência da introdução de Mingau*2) + (Frequência da introdução de Sopa*2) + (Frequência da introdução de Comida Caseira*2) + (Frequência da introdução de Biscoito*2) *100\}. O escore da alimentação complementar poderia, assim, variar de 1.400 (escore mínimo: introdução de todos os alimentos antes dos 2 meses de idade) até 5.600 (escore máximo: introdução de todos os alimentos após os 6 meses). Dessa forma, um escore baixo da alimentação complementar indicaria que houve introdução precoce de alimentos. 
Os modelos de regressão linear múltipla foram ajustados pelas seguintes covariáveis: idade do pré-escolar e idade materna. A covariável sexo do escolar foi introduzida no modelo final como uma variável de ajuste em função de sua distribuição limítrofe entre as categorias do excesso de peso $(p=0,195)$. As análises estatísticas foram realizadas no programa estatístico Statistical Analysis System (SAS) versão 8.2.14

\section{Resultados}

Os pré-escolares avaliados tinham em média 3,3 ( \pm 0,91) anos de idade, $15,9( \pm 3,10) \mathrm{kg}$ e 99,3 $( \pm 7,82) \mathrm{cm}$. As mães dos pré-escolares apresentaram em média 29,1 $( \pm 6,59)$ anos de idade, 8,1 $( \pm 3,05)$ anos de escolaridade e 2,0 ( $\pm 1,16)$ filhos (tabela 1). Do total de 126 crianças avaliadas antropometricamente, $36(28,6 \%)$ apresentavam algum grau de excesso de peso (tabela 1$)$.

Entre os pré-escolares, $64(50,8 \%)$ eram do sexo masculino e 62 (49,2\%) do sexo feminino (tabela 2). Entre os meninos e as meninas, respectivamente, $15(23,4 \%)$ e $21(33,9 \%)$ apresentaram excesso de peso (tabela 2), mas a diferença da prevalência entre os sexos não foi significativa $(p=0,195)$.

Nota-se, na tabela 2, que não há diferença estatisticamente significativa entre as características antropométricas e sociodemográficas dos pré-escolares segundo o estado nutricional (com e sem excesso de peso). Entretanto, a prevalência de aleitamento materno exclusivo diferiu segundo o estado nutricional dos pré-escolares com excesso de peso $(p=0,028)$. Em relação à introdução de alimentos (suco/fruta; mamadeira, legumes, sopa, mingau, comida caseira e biscoito), não houve diferença significativa entre as categorias do estado nutricional (com e sem excesso de peso).

$\mathrm{Na}$ análise de regressão linear univariada, as variáveis idade do pré-escolar $(p<0,001)$ e idade materna $(p=0,003)$ mostraram-se associadas ao IMC para idade (tabela 3). As demais covariáveis não apresentaram associação $(p>0,20)$ e por isso não foram consideradas como variáveis de ajuste, exceto a variável "sexo do pré-escolar", que foi considerada confundimento em função da sua distribuição limítrofe $(p<0,20)$ entre as categorias do estado nutricional (com e sem excesso de peso).

A tabela 4 apresenta os modelos multivariados do IMC para idade segundo as variáveis explicativas: aleitamento materno, introdução dos alimentos no primeiro ano e escore da alimentação complementar. Dentre os alimentos listados, verificou-se associação positiva do IMC para idade com o mingau $(\beta=0,0080$; IC95\% $=0003-0,015 ; p=0,043)$ e associação negativa com o escore de alimentação complementar $(\beta=-0,001$; IC95\% $=-0,002--0003 ; p=0,016)$. 
Tabela 4. Regressão linear multivariada do índice de massa corporal e aleitamento materno, introdução de alimentos e escore da alimentação complementar. Rio de Janeiro-RJ, 2012.

\begin{tabular}{lcccc}
\hline \multicolumn{1}{c}{ Variáveis } & $\beta$ & $\mathrm{IC95 \%}$ & $\mathrm{R}^{2}$ & Valor de $p$ \\
\hline $\begin{array}{l}\text { Aleitamento materno exclusivo } \\
\text { Introdução de alimentos }\end{array}$ & 0,166 & $-0,215-0,548$ & 0,412 & 0,388 \\
Frutas/Suco & 0,012 & $-0,169-0,193$ & 0,408 & 0,898 \\
Legumes & $-0,060$ & $-0,241-0,121$ & 0,435 & 0,509 \\
Mamadeira & 0,0002 & $-0,012-0,013$ & 0,395 & 0,977 \\
Mingau & 0,008 & $0,0003-0,015$ & 0,386 & 0,043 \\
Sopa & $-0,063$ & $-0,241-0,115$ & 0,412 & 0,483 \\
Comida Caseira & $-0,047$ & $-0,228-0,135$ & 0,412 & 0,612 \\
Biscoito & $-0,137$ & $-0,313-0,040$ & 0,427 & 0,128 \\
Escore $^{\text {†े }}$ & $-0,001$ & $-0,002--0003$ & 0,465 & 0,016 \\
\hline
\end{tabular}

Modelos ajustados pelas variáveis idade do pré-escolar e idade materna.

* Escore da alimentação complementar: (Frutas/Suco*2) + (Legumes*2) + (Mamadeira*2) + (Mingau*2) + $($ Sopa $* 2)+($ Comida Caseira*2) $+($ Biscoito*2) $* 100$.

\section{Discussão}

No presente estudo, 28,6\% das crianças avaliadas apresentaram algum grau de excesso de peso. A ausência de aleitamento materno exclusivo e a introdução precoce de mingau se mostraram fortemente associadas a esse desfecho, assim como a relação negativa do escore da alimentação complementar com o IMC para idade. Ou seja, quanto mais tarde a introdução dos alimentos, menor foi o IMC para idade entre os pré-escolares.

A prevalência da obesidade infantil aumentou nas últimas décadas em todo o mundo, sendo caracterizada como uma verdadeira epidemia. ${ }^{15}$ No Brasil, a Pesquisa Nacional sobre Demografia e Saúde ${ }^{16}$ divulgada em 2008 demonstrou que 7,8\% das crianças menores de cinco anos apresentavam algum grau de excesso de peso. Dentre os motivos para essa situação, foram assinalados a introdução precoce de alimentos, o consumo de alimentos industrializados e o sedentarismo. ${ }^{17}$

A literatura é consensual ao reconhecer que as fases iniciais, sobretudo durante o primeiro ano de vida, são sensíveis a alterações nutricionais e metabólicas, e que podem determinar efeitos na saúde da criança a curto e longo prazos. ${ }^{11,18,19}$ Em relação ao excesso de peso, achados 
epidemiológicos sugerem que a prática do aleitamento materno pode representar um fator de proteção contra obesidade na infância e adolescência. ${ }^{5,18-21}$ Nesse sentido, acredita-se que diversos fatores estejam relacionados com a prevenção do desenvolvimento de obesidade, dentre os quais se destacam: a especificidade do leite humano e a influência de fatores ambientais e comportamentais, como nível socioeconômico, escolaridade materna, padrão alimentar e prática de atividade física. ${ }^{4}$

Em um estudo de meta-análise, com o objetivo de verificar a associação entre práticas alimentares na infância e a obesidade em idades posteriores, Owen et al..$^{19}$ observaram que o aleitamento materno apresentou efeito protetor contra a obesidade, quando comparado ao aleitamento artificial $\left(\mathrm{OR}=0,87\right.$; IC 95\% = 0,85 - 0,90). Mais recentemente, Messiah et al., ${ }^{22} \mathrm{em}$ estudo americano de base populacional, avaliaram a influência da amamentação, da introdução precoce de alimentos sólidos, fumo durante a gravidez e baixo-peso ao nascer como fatores de risco para doenças cardiovasculares entre pré-escolares com idade entre 3-6 anos. Entre os resultados apresentados, os autores mostraram a amamentação como um fator de proteção para obesidade infantil (OR 0,43, 95\% CI, 0,27-0,69).

No Brasil, Siqueira \& Monteiro ${ }^{4}$ analisaram a associação entre exposição ao aleitamento materno na infância e a obesidade na idade escolar em crianças de famílias brasileiras de alto nível socioeconômico. Os autores concluíram que a ocorrência de obesidade em crianças que nunca foram amamentadas foi duas vezes superior a das demais crianças (OR=2,06; IC 95\%: 1,02; 4,16).

No presente estudo, outros fatores que influenciaram o estado nutricional dos pré-escolares foram: idade materna, uma vez que quanto maior a idade materna, maior o IMC para idade da criança; e introdução precoce do mingau, que apresentou associação positiva com o IMC para idade. Ademais, o escore da alimentação complementar associou-se negativamente com o IMC para idade. Estudo de revisão realizado por Monte \& Giugliani ${ }^{9}$ aponta os riscos da alimentação complementar precoce e assinala os poucos avanços da promoção da alimentação complementar na prática clínica e nutricional.

Quanto à idade materna, Guimarães et al. ${ }^{23}$ também encontraram maior ocorrência de excesso de peso entre escolares filhos de mães mais velhas. Patterson et al. ${ }^{24}$ corroboram que o aumento da prevalência de obesidade está associado com o aumento da idade materna, em casuística com dados de meninas americanas da faixa etária de 9-10 anos. Os autores discutem a possibilidade de mães mais velhas tenderem a proteger mais os filhos e, consequentemente, oferecerem maiores quantidades de alimentos.

Os resultados obtidos no estudo longitudinal conduzido por Li et al. ${ }^{20}$ nos Estados Unidos, com 1.899 lactentes, mostraram ganho ponderal maior entre crianças em aleitamento artificial durante o primeiro ano de vida. Outro estudo, conduzido com 7.798 crianças irlandesas com nove 
anos de idade, concluiu que a amamentação, por um período entre 13-25 semanas, foi associada com a redução de $38 \%(p<0,05)$ do risco de obesidade aos nove anos de idade, enquanto essa prática por período igual ou superior a 26 semanas esteve associada com uma redução de $51 \%$ ( $p$ $<0,01)^{25}$ do mesmo risco.

Em estudo longitudinal de coorte de nascimento realizado por Bergmann et al., ${ }^{6}$ com 918 crianças acompanhadas até os seis anos de idade, os autores constataram maior proporção de IMC elevado aos seis meses de idade para os lactentes amamentados com fórmulas infantis. E assinalaram que a obesidade minimamente duplicou dos quatro para os cinco e seis anos de idade. $\mathrm{O}$ estudo de Koletzko et al. ${ }^{26}$ evidenciou, entre as crianças que receberam fórmulas com maiores teores proteicos, valores significativamente mais elevados de IMC, quando comparadas às crianças amamentadas e recebendo fórmulas com menores conteúdos proteicos ou dentro dos limites preconizados pelo Codex Alimentarius.

Evidências crescentes ressaltam que a introdução precoce de alimentos inadequados, como leite de vaca integral, alimentos com baixa biodisponibilidade de micronutrientes; ou ainda, a oferta insuficiente de frutas, verduras e legumes, paralelamente ao consumo excessivo de carboidratos simples, lipídios e sódio, contribuem para a formação de hábitos alimentares não saudáveis, ${ }^{26}$ que repercutem negativamente sobre o estado nutricional e, consequentemente, sobre a saúde dos pré-escolares.

A Organização Mundial da Saúde ${ }^{13}$ reconhece a prática do aleitamento materno exclusivo nos seis primeiros meses de vida, e de forma complementar até os dois anos, e a introdução da alimentação complementar oportuna e equilibrada como estratégias de saúde pública eficazes para a redução do risco do desenvolvimento de doenças crônicas não transmissíveis. ${ }^{2}$

Vale destacar a necessidade de desenvolver um acompanhamento rotineiro dessa população, tanto para monitorar o estado nutricional dos pré-escolares, como também para orientar as crianças e as famílias sobre a prevenção da obesidade, um problema de saúde pública atual e relevante. A falta de informações sobre as condições ao nascer limitou a observação de associações entre essa informação e os eventos estudados, assim como o desenho transversal do estudo impossibilitou estabelecer relações causais.

Embora sem significância, muito provavelmente em função do tamanho amostral, o presente estudo indicou uma introdução de alimentos elevada antes dos quatro meses de idade, independentemente do excesso de peso. Essa concorrência do aleitamento materno precoce, além de ser inadequada e pouco saudável, pode contribuir para o risco de desenvolvimento de doenças futuras, dentre as quais se destacam as cardiovasculares agravadas pelo excesso de peso. ${ }^{15,19}$ 
Hoje a realização do Direito Humano à Alimentação Adequada ${ }^{27}$ está prevista na Constituição Brasileira, ${ }^{28}$ e a promoção da alimentação adequada e saudável é uma das diretrizes da atual Política Nacional de Alimentação e Nutrição (PNAN), ${ }^{1}$ sendo uma das estratégias implementadas pela Política Nacional de Promoção da Saúde ${ }^{29}$ para promover a qualidade de vida e reduzir os danos à saúde.

A PNAN, ${ }^{1}$ inserida no contexto da alimentação adequada e saudável, reconhece o aleitamento materno como a primeira prática alimentar adequada, e ressalta a importância do apoio da rede de atenção à saúde, tanto no incentivo do aleitamento materno exclusivo até os seis meses, como para a orientação da alimentação complementar saudável, que deve ocorrer até os dois anos de idade. A vigilância alimentar e nutricional (VAN), terceira diretriz contida na PNAN, pode ser empregada como ferramenta estratégica para o adequado monitoramento do estado nutricional de pré-escolares.

Assinala-se, por fim, a importância de investigações longitudinais prospectivas que avaliem o impacto da alimentação complementar no primeiro ano de vida sobre o estado nutricional de pré-escolares e na incidência futura de doenças cardiovasculares. Diante das evidências atuais, sugere-se a valorização da amamentação exclusiva nos seis primeiros meses de vida e a elaboração de estratégias para promoção da alimentação complementar saudável.

\section{Referências}

1. Brasil. Ministério da Saúde. Secretaria de Atenção à Saúde. Departamento de Atenção Básica. Política Nacional de Alimentação e Nutrição/Ministério da Saúde. Secretaria de Atenção à Saúde. Departamento de Atenção Básica - Brasília: Ministério da Saúde, 2012.

2. Brasil. Ministério da Saúde. Secretaria de atenção à saúde. Departamento de atenção básica. Saúde da criança: nutrição infantil: aleitamento materno e alimentação complementar. Brasília: Ministério da Saúde, 2009.

3. Balaban G, Silva GA. Protective effect of breastfeeding against childhood obesity. J Pediatr 2004; 80:7-16.

4. Siqueira RS, Monteiro CA. Breastfeeding and obesity in school-age children from families of high socioeconomic status. Rev Saude Publica 2007; 41:5-12.

5. Simon VG, Souza JM, Souza SB. Breastfeeding, complementary feeding, overweight and obesity in pre-school children. Rev Saude Publica 2009; 43:60-9.

6. Bergmann RL, Kries R, Richter OB, Dudenhausen JW, Wahn U. Early determinants of childhood overweight and adiposity in a birth cohort study: role of breast-feeding. Inter J of Obesity 2003; 27:162-172. 
7. Metzger MW, McDade TW. Breastfeeding as Obesity Prevention in the United States:A Sibling Difference Model. American J of Human Biology 2010; 22:291-296.

8. Brasil - Ministério da Saúde. Secretaria de atenção à saúde - departamento de ações programáticas e estratégicas. II Pesquisa de Prevalência de Aleitamento Materno nas Capitais Brasileiras e Distrito Federal. Brasília: Ministério da Saúde, 2009.

9. Monte CM, Giugliani ER. Recommendations for the complementary feeding of the breastfed child. J Pediatr 2004;80 (Suppl 5):131-41.

10. Waterland RA, Garza C. Potential mechanisms of metabolic imprinting that lead to chronic disease. Am J Clin Nutr 1999;69:179-97.

11. Caetano MC, Ortiz TT, Silva SG, Souza FI, Sarni SO. Complementary feeding: inappropriate practices in infants. J Pediatr 2010; 86:196-201.

12. Lohman TG. Guest Reviewers Advances in Body Composition Assessment. Current Issues in Exercise Science (Monograph No. 3). Champaign: Human Kinetics Publishers; 1992.

13. World Health Organization. WHO Child Growth Standards: length/height-for-age, weight-for-age, weight-for-length, weight-for-height and body mass index-for-age. Geneva: WHO; 2006.

14. Statistical Analysis System. SAS/ETS Software: changes and enhancements, release 8.2. Cary: SAS Institute Inc; 2000.

15. De Onis M, Blössner M, Borghi E. Global prevalence and trends of overweight and obesity among preschool children. Am J Clin Nutr 2010;92:1257-64.

16. Brasil - Ministério da Saúde. Secretaria de ciência, tecnologia e insumos estratégicos - departamento de ciência e tecnologia. Pesquisa Nacional de Demografia e Saúde da Criança e da Mulher - PNDS 2006 [Relatório]. Brasília: Ministério da Saúde, 2008.

17. Barreto AC, Brasil LM, Maranhão HS. Sobrepeso: uma nova realidade no estado nutricional de préescolares de Natal, RN. Rev Assoc Med Bras 2007;53:311-6.

18. Butte NF. The role of breastfeeding in obesity. Pediatr Clin North Am 2001;48:189-98.

19. Owen CG, Martin RM, Whincup PH, Smith GD, Cook DG. Effect of infant feeding on the risk of obesity across the life course: a quantitative review of published evidence. Pediatrics 2005;115:1367-77.

20. Li R, Fein SB, Chen J, Grummer-Strawn LM.Why mothers stop breastfeeding: mothers' self-reported reasons for stopping during the first year. Pediatrics 2008;122 (Suppl 2):S69-76.

21. Scott JA, Ng SY, Cobiac L. The relationship between breastfeeding and weight status in a national sample of Australian children and adolescents. BMC Public Health 2012;12:107.

22. Messiah SE, Arheart KL, Lipshultz SE, Bandstra ES, Miller TL. Perinatal Factors Associated with Cardiovascular Disease Risk among Preschool-Age Children in the United States: An Analysis of 1999-2008 NHANES Data. Int J Pediatr 2012;2012:157-237.

23. Guimarães LV, Barros MB, Martins MS, Duarte EC. Factors associated with overweight in schoolchildren. Rev Nutr 2006;19:5-17. 
24. Patterson ML, Stern S, Crawford PB, McMahon RP, Similo SL, Schreiber GB et al. Sociodemographic factors and obesity in preadolescent black and white girls: NHLBI's Growth and Health Study. J Natl Med Assoc 1997;89:594-600.

25. McCrory C, Layte R. Breastfeeding and risk of overweight and obesity at nine-years of age. Soc Sci Med 2012;75:323-30.

26. Koletzko B, von Kries R, Monasterolo RC, Subías JE, Scaglioni S, Giovannini M et al. Infant feeding and later obesity risk. Adv Exp Med Biol 2009;646:15-29.

27. Burity V, Franceschini T, Valente F, Recine E, Leão M, Carvalho MF. Direito humano à alimentação adequada no contexto da segurança alimentar e nutricional. Brasília, DF: ABRANDH, 2010. 204p.

28. Brasil. Constituição Federal Brasileira de 1988.

29. Brasil. Ministério da Saúde. Secretaria de Vigilância em Saúde. Política nacional de promoção da saúde / Ministério da Saúde, Secretaria de Atenção à Saúde. - Brasília: Ministério da Saúde, 2006. 60 p. - (Série B. Textos Básicos de Saúde).

Recebido: $11 / 5 / 2014$

Revisado: 06/8/2014

Aprovado: 02/9/2014 